\title{
First-principles Calculation of Excited State Spectra in QCD
}

\author{
Jozef J. Dudek*,†, Robert G. Edwards*, Michael J. Peardon**, David \\ G. Richards* and Christopher E. Thomas* \\ * Jefferson Laboratory, 12000 Jefferson Avenue Suite \#1, Newport News, VA 23606, USA \\ ${ }^{\dagger}$ Department of Physics, Old Dominion University, Norfolk, VA 23529, USA \\ ** School of Mathematics, Trinity College, Dublin 2, Ireland
}

\begin{abstract}
Recent progress at understanding the excited state spectra of mesons and baryons is described. I begin by outlining the application of the variational method to compute the spectrum of QCD, and then present results for the excited meson spectrum, with continuum quantum numbers of the states clearly delineated. I emphasise the need to extend the calculation to encompass multihadron contributions, and describe a recent calculation of the $I=2 \pi \pi$ energy-dependent phase shifts as a precursor to the study of channels with resonant behavior. I conclude with recent results for the low lying baryon spectrum, and the prospects for future calculations.
\end{abstract}

Keywords: Spectroscopy, Lattice QCD

PACS: 12.38.Gc,11.15.Ga,14.20.Gk,14.40.Rt

In order to really understand QCD and hence test whether it is the complete theory of the strong interaction, we must know the spectrum of mesons and baryons that it implies and test those spectra against high quality data. The three valence quarks of baryons are emblematic of the non-abelian nature of QCD, and the determination of the $N^{*}$ spectrum is thus a key experimental effort. The observed spectrum of QCD provides little direct evidence of the presence of the gluons. However, QCD admits the possibility of exotic mesonic states of matter in which the gluonic degrees of freedom are explicitly exhibited. The search for such states will be an important component of the upgraded JLab@12GeV. To capitalize on these experimental efforts, the need to predict and understand the spectrum from first principles using lattice QCD is clear.

A comprehensive picture of resonances within lattice $\mathrm{QCD}$ requires that we go beyond a knowledge of the ground state mass in each channel, and obtain the masses of the lowest states of a given quantum number. This we can accomplish through the use of the variational method $[1,2]$. Rather than measuring a single correlator $C(t)$, we determine a matrix of correlators

$$
C_{i j}(t)=\sum_{\vec{x}} \sum_{\vec{y}}\left\langle O_{i}(\vec{x}, t) O_{j}^{\dagger}(\vec{y}, 0)\right\rangle,
$$

where $\left\{O_{i} ; i=1, \ldots, N\right\}$ are a basis of interpolating operators with given quantum numbers. We then solve the generalized eigenvalue equation $C(t) u=\lambda\left(t, t_{0}\right) C\left(t_{0}\right) u$ to obtain a set of real (ordered) eigenvalues $\lambda_{n}\left(t, t_{0}\right)$, where $\lambda_{0} \geq \lambda_{1} \geq \cdots \geq \lambda_{N-1}$. The behavior of these eigenvalues at large Euclidean time yields the different energies

The (hyper-) cubic lattice does not possess the full rotational symmetry of the continuum. Thus in a lattice calculation, states at rest are classified not according to the spin 
$\left(J, J_{z}\right)$, but rather according to the irreducible representations (irreps) of the symmetry group of the cube; for states of higher spin, the different continuum degrees of freedom are distributed across several lattice irreps. In the case of the meson spectrum, we are able to reliably identify the spins of the (single-hadron) states by employing operators of definite spin in the continuum limit. Our ability to calculate correlation functions efficiently for such a large basis of operators has been greatly advanced through the development of a new method, "distillation"[3] and its decendents[4], for the construction of the interpolating operators.

\section{EXCITED MESON SPECTRUM}

The reduced cubic symmetry imposed by the lattice has proved to be a formidable barrier to the assignment of continuum spins to energy eigenstates, and hence to the interpretation of lattice calculations of the spectrum. We have found that the judicious choice of interpolating operators is crucial to the identification of the meson spins. Our starting point is a set of interpolating operators

$$
\mathscr{O}=\sum_{\vec{x}} \bar{\psi}(\vec{x}) \Gamma \overleftrightarrow{D}_{i} \overleftrightarrow{D}_{j} \ldots \psi(\vec{x})
$$

where $\overleftrightarrow{D}$ is a discretized covariant derivative, and $\psi(\bar{\psi})$ a quark (antiquark) field. From this set, we can construct a basis of interpolating operators $\mathscr{O}^{J, M}$ that in the continuum have definite spin,

$$
\left\langle 0\left|\mathscr{O}^{J M}\right| J^{\prime}, M^{\prime}\right\rangle=Z^{J} \delta_{J, J^{\prime}} \delta_{M, M^{\prime}}
$$

It is these operators that are now subduced to the lattice irreducible representations:

$$
\mathscr{O}_{\Lambda, \lambda}^{[J]}=\sum_{M} S_{\Lambda, \lambda}^{J, M} \mathscr{O}^{J, M}
$$

where $\Lambda, \lambda$ denote the lattice irrep. and its row respectively, and $S_{\Lambda, \lambda}^{J, M}$ are calculable coefficients. Whilst the resulting operators $\mathscr{O}_{\Lambda, \lambda}^{[J]}$ interpolate to any state whose spin is subduced to the irrep. $\Lambda$, we find that in practice the operator interpolates strongly to states of spin $J$, and that the resulting overlaps $Z^{J}$ are common across the different subductions; the method is described in detail in ref. [5]. Thus the operators $\mathscr{O}_{\Lambda, \lambda}^{[J]}$ retain a strong memory of their continuum antecedents, and this property enables us to identify the spins of the states for the effectively single-hadron states we have observed in the following calculation.

The availability of the large ensembles of $N_{f}=2+1$ anisotropic clover lattices[6,7], together with the efficient operator-construction admitted by "distillation", has enabled us to compute the isovector meson spectrum for states composed of the light and strange quarks $[8,5]$, at pion masses down to around $400 \mathrm{MeV}$. Masses of highly excited states have been calculated, with the quantum numbers reliably delineated, including for the first time in a lattice calculation states of spin 4; this is illustrated in Figure 1 for the case of the three degenerate quark flavors. Figure 2 summarizes the results on 


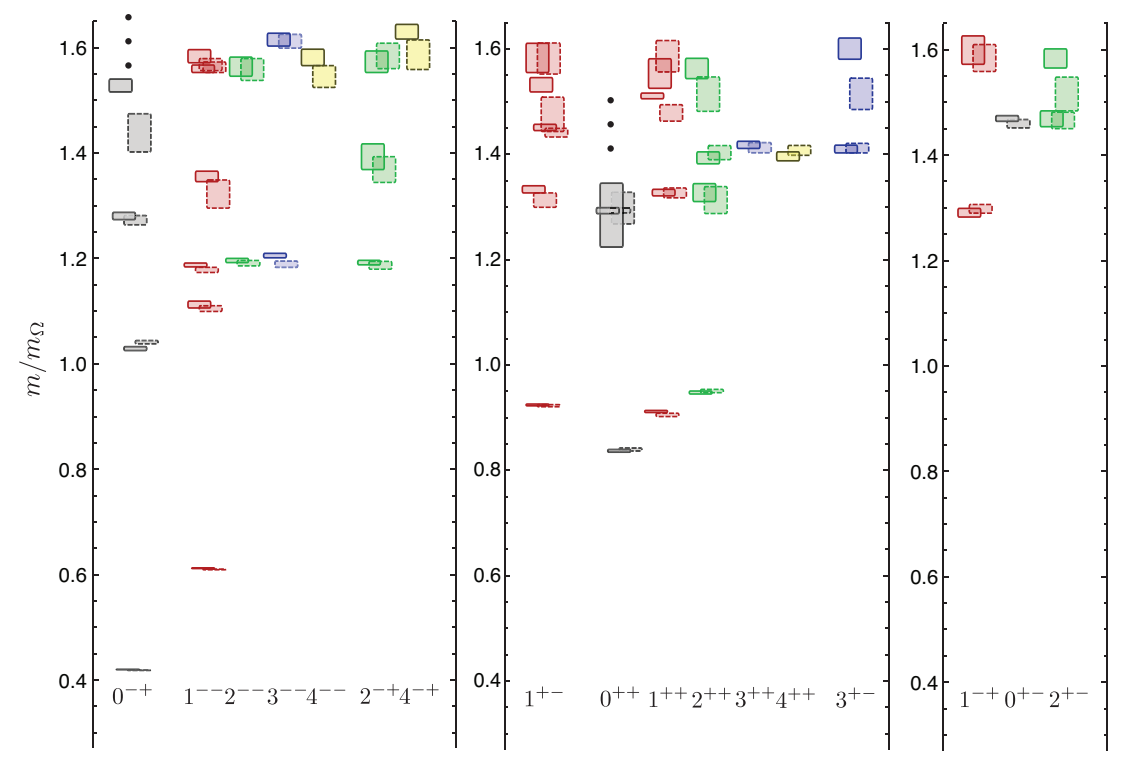

FIGURE 1. Spin-identified spectrum of isovector (octet) mesons in a calculation with three degenerate flavors of quarks of mass around that of the strange quark[5]. The calculation is performed at two spatial volumes: $16^{3}$ (solid) and $20^{3}$ (dashed) spectra agree well. Ellipses indicate that there are heavier states with a given $J^{P C}$ but that they are not well determined in this calculation. Note that the right-most panel corresponds to states of exotic quantum numbers.

exotic states and compares those results with previous lattice QCD results from Refs. $[9,10,11,12,13,14]$. Furthermore, we see indications of hybrid states, that is those with an excited gluonic field, that have conventional, non-exotic quantum numbers.

The experimental implication of these results is important: there are likely mesons of exotic quantum numbers in a mass region accessible to the GlueX experiment at JLab12GeV. A notable feature of our calculation is that we see little evidence for the two-particle states that must be in the spectrum. In order to improve our sensitivity to such states, we believe it necessary to expand our basis of interpolating operators to include those with larger numbers of fermion fields.

\section{$I=2 \pi \pi$ PHASE SHIFT}

The analysis of channels which display resonant behavior, both in the meson and in the baryon sector, is a major challenge. Lüscher's method provides a means of relating the discrete spectrum of states in a finite volume to the energy-dependent phase shift[15]. To explore the feasibility of the system, we look at $I=2 \pi \pi$ scattering, a channel that does not exhibit resonant behavior.

We use as the basis of interpolating operators back-to-back pions at specified relative momentum, with the total momentum of the two-particle system zero[16]:

$$
\mathscr{O}_{\pi \pi}^{\Gamma, \gamma}(|\vec{p}|)=\sum_{m} S_{\Gamma, \gamma}^{\ell, m} \sum_{\hat{p}} Y_{\ell}^{m}(\hat{p}) \mathscr{O}_{\pi}(\vec{p}) \mathscr{O}_{\pi}(-\vec{p})
$$




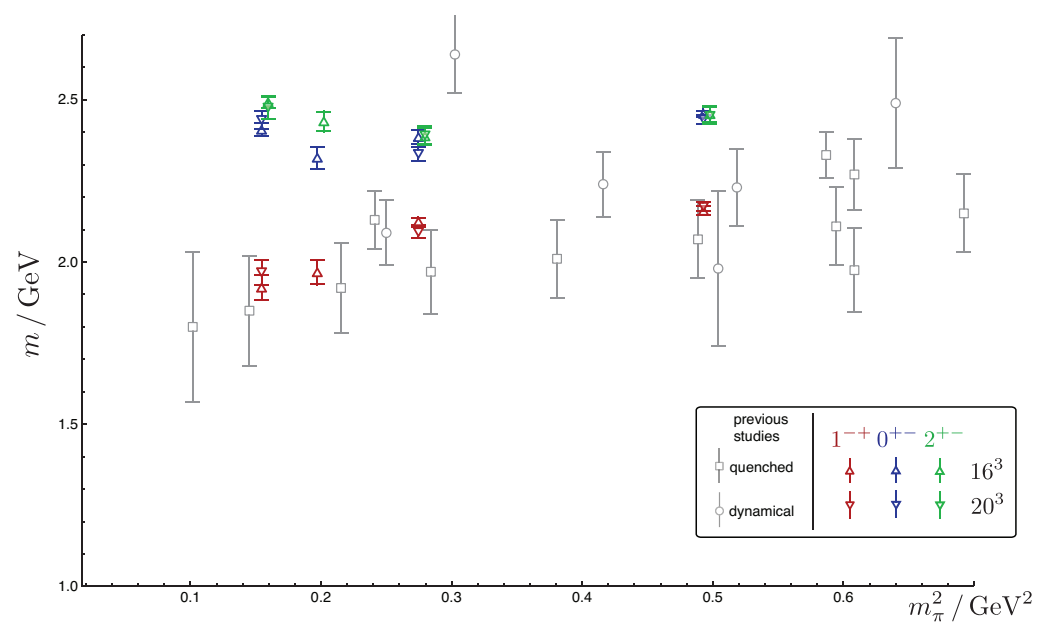

FIGURE 2. Summary of extracted isovector exotic states. For comparison $1^{-+}$results from Refs. $[9,10,11,12,13,14]$ are also plotted.

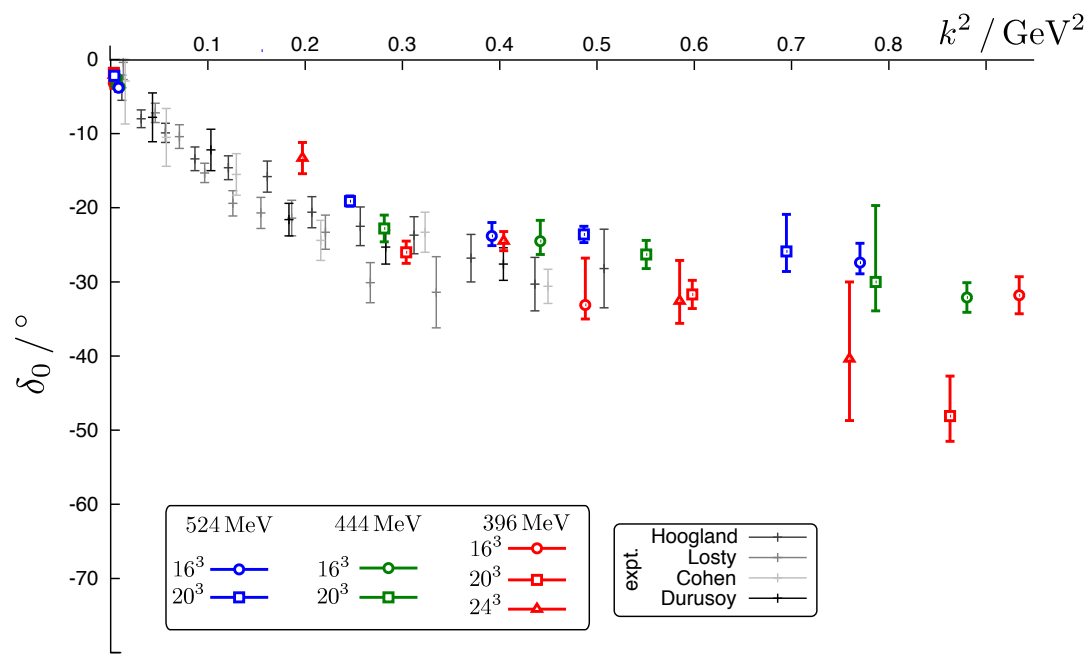

FIGURE 3. The $I=2 \pi \pi$ phase shift for $l=0$, at various pion masses[16]. Also shown are the experimental data[17, 18, 19, 20].

where the coefficients $S_{\Gamma, \gamma}^{\ell, m}$ project operators of definite $l$ into definite irreps. $\Gamma$, with row $\gamma$. Note that momenta are those allowed by the cubic symmetry of the boundary. Once again, "distillation" provides a means of efficiently computing the correlators from these operators.

The $l=0$ and $l=2$ phase shifts are shown in Figures 3 and 4, respectively. We observe no significant pion-mass dependence, with results in reasonable agreement with experiment at low scattering momenta. Most importantly, however, this calculation lays the groundwork for the investigation of scattering in channels which do exhibit resonant behavior. 


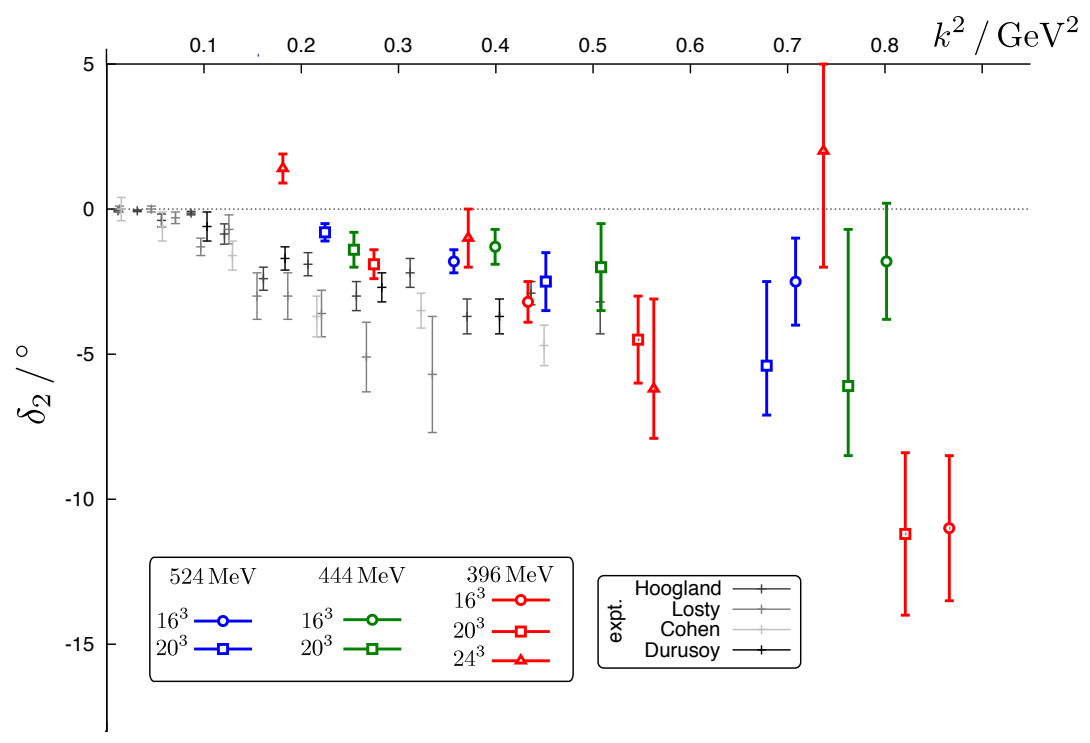

FIGURE 4. The $I=2 \pi \pi$ phase shift for $l=2$, at various pion masses[16]. Also shown are the experimental data[17, 18, 19, 20].

\section{BARYON RESONANCES IN LATTICE QCD}

In this talk, I have focused on meson spectroscopy both for its inherent interest and as an exemplar of the methodology. An important goal in exploring baryons is attempting to discern the effective degrees of freedom that describe the spectrum; the search for so-called "missing resonances" focuses on whether the spectrum can be well described by a quark model, or whether an effective theory with fewer degrees of freedom, such as a quark-diquark picture, provides a more faithful description of the baryon spectrum.

The procedure for constructing a very general basis of interpolating operators has been detailed[21,22]. The spectra of the Nucleon, $\Delta$ and $\Omega$ baryons on the $N_{f}=2 \oplus 1$ flavor lattices, have been calculated at three values of the light-quark masses corresponding to $m_{\pi}=392,438$ and $521 \mathrm{MeV}$ [23], once again using the "distillation" method. For the $\Omega$, the experimental spin-parity assignment is only known for the lowest-lying state, and the determination of the spin-parity assignments is thus an important predictive opportunity for lattice QCD. The task of identifying the quantum numbers of the states will be greatly facilitated through the use of operators subduced from their continuum counterparts, in analogy with the meson calculations above[24]. Finally, we again note the need to include multi-hadron operators in our operator basis to resolve the multihadron states present in the spectrum.

\section{SUMMARY}

I have shown in this talk how the use of the variational method, using a large basis of interpolating operators with known continuum behaviours, is enabling the calculation of the excited spectrum of QCD, with the quantum numbers of single hadron states identified. The $I=2 \pi \pi$ energy-dependent phase shift has been computed, laying the 
groundwork for the study of states displaying resonant behavior. Future work will concentrate on the amalgam of these developments, the calculation of the isoscalar spectrum, and the progression of calculations to the physical light-quark masses.

\section{ACKNOWLEDGMENTS}

I am grateful to my colleagues in the Hadron Spectrum Collaboration, whose work is presented here. Supported by the U.S. DOE Contract No. DE-AC05-06OR23177 under which Jefferson Science Associates, LLC operates the Thomas Jefferson National Accelerator Facility. The Chroma software suite [25] was used to perform this work on clusters at Jefferson Laboratory and Fermilab using time awarded under the USQCD Initiative. The U.S. Government retains a non-exclusive, paid-up, irrevocable, worldwide license to publish or reproduce this manuscript for U.S. Government purposes.

\section{REFERENCES}

1. C. Michael, Nucl. Phys. B259, 58 (1985).

2. M. Luscher, and U. Wolff, Nucl. Phys. B339, 222-252 (1990).

3. M. Peardon, et al., Phys. Rev. D80, 054506 (2009), 0905.2160.

4. C. Morningstar, et al. (2011), these proceedings.

5. J. J. Dudek, R. G. Edwards, M. J. Peardon, D. G. Richards, and C. E. Thomas, Phys. Rev. D82, 034508 (2010), 1004.4930.

6. R. G. Edwards, B. Joo, and H.-W. Lin, Phys. Rev. D78, 054501 (2008), 0803.3960.

7. H.-W. Lin, et al., Phys. Rev. D79, 034502 (2009), 0810.3588.

8. J. J. Dudek, R. G. Edwards, M. J. Peardon, D. G. Richards, and C. E. Thomas, Phys. Rev. Lett. 103, 262001 (2009), 0909.0200.

9. C. Bernard, et al., Phys. Rev. D68, 074505 (2003), hep-lat/ 0301024.

10. C. W. Bernard, et al., Phys. Rev. D56, 7039-7051 (1997), hep-lat/9707008.

11. P. Lacock, and K. Schilling, Nucl. Phys. Proc. Suppl. 73, 261-263 (1999), hep-lat/ 9809022.

12. P. Lacock, C. Michael, P. Boyle, and P. Rowland, Phys. Lett. B401, 308-312 (1997), hep-lat/ 9611011.

13. C. McNeile, and C. Michael, Phys. Rev. D73, 074506 (2006), hep-lat / 0603007.

14. J. N. Hedditch, et al., Phys. Rev. D72, 114507 (2005), hep-lat/ 0509106.

15. M. Luscher, Nucl. Phys. B354, 531-578 (1991).

16. J. J. Dudek, R. G. Edwards, M. J. Peardon, D. G. Richards, and C. E. Thomas (2010), 1011.6352.

17. W. Hoogland, et al., Nucl. Phys. B126, 109 (1977).

18. D. H. Cohen, T. Ferbel, P. Slattery, and B. Werner, Phys. Rev. D7, 661 (1973).

19. M. J. Losty, et al., Nucl. Phys. B69, 185-204 (1974).

20. N. B. Durusoy, et al., Phys. Lett. B45, 517-520 (1973).

21. S. Basak, et al., Phys. Rev. D72, 074501 (2005), hep-lat/ 0508018.

22. S. Basak, et al., Phys. Rev. D72, 094506 (2005), hep-lat/ 0506029.

23. J. Bulava, et al., Phys. Rev. D82, 014507 (2010), 1004.5072.

24. R. G. Edwards, et al. (2010), in preparation.

25. R. G. Edwards, and B. Joo, Nucl. Phys. Proc. Suppl. 140, 832 (2005), hep-lat / 0409003. 